\title{
Perception of bar velocity in resistance training: accuracy levels within and between exercises
}

Submission Type: Original article

Authors: Mahmood Sindiani ${ }^{1 \dagger}$, Amit Lazarus ${ }^{2 \dagger}$ Antonio Dello Iacono $^{3}$, and Israel

Halperin $^{4,5 *}$

\author{
Affiliations: \\ ${ }^{1}$ Life Science Department, The Academic College at Wingate, Netanya, Israel \\ ${ }^{2}$ Faculty of Social Sciences, Department of Sociology and Anthropology, Tel-Aviv \\ University, Tel-Aviv, Israel \\ ${ }^{3}$ School of Health and Life Sciences, University of the West of Scotland, Hamilton, United \\ Kingdom, \\ ${ }^{4}$ School of Public Health, Sackler Faculty of Medicine, Tel-Aviv University, Tel-Aviv, \\ Israel \\ ${ }^{5}$ Sylvan Adams Sports Institute, Tel Aviv University, Tel-Aviv, Israel \\ ${ }^{\dagger}$ Mahmood Sindiani and Amit Lazarus are joint first authors as they have contributed \\ equally to this manuscript. \\ *Corresponding author: Israel Halperin, School of Public Health, Sackler Faculty of \\ Medicine, Tel-Aviv University, Tel-Aviv, Israel. \\ Phone: +972-523-469-590 \\ Email: ihalperin@tauex.tau.ac.il
}

Author agreement statement: We the authors agree to the sharing of this preprint onSportR $\chi$ iv. 
Cite as: Sindiani M, Lazarus A, Della-Iacono A, Halperin I (2020). Perception of bar velocity in resistance training: accuracy levels within and between exercises. Pre-print DOI: 10.31236/osf.io/mnyv5

Word count: 3485

\begin{abstract}
Background: Velocity-based training is a method used to monitor resistance-training programs based on repetition velocities measured with tracking devices. Since velocity measuring devices can be expensive and impractical, trainee's perception of velocity (POV) may be used as a possible substitute.
\end{abstract}

Methods: Twenty resistance-trained males first completed 1RM tests in the bench-press and squat. Then, in three counterbalanced sessions, participants completed four sets of eight repetitions in both exercises using 60\%1RM (two-sessions) or 70\%1RM. Starting from the second repetition, participants reported their POV of each repetition as a percentage of the first repetition. Accuracy of POV was calculated as the difference between POV and the velocity measured with a linear-encoder.

Results: Three key findings emerged. First, the absolute error in the bench-press and squat was $\approx 5.8$ percentage-points in the second repetition, and increased to 13.2 and 16.7 percentage-points, respectively, by the eighth repetition. Second, subjects reduced the absolute error in the second $60 \% 1 \mathrm{RM}$ session compared to the first by $\approx 1.7$ in both exercises $(\mathrm{p} \leq 0.007)$. Third, participants were 4.2 times more likely to underestimate velocity in the squat compared to the bench-press. 
Conclusion: The gradual increments in the absolute error suggests that POV may be better suited for sets of fewer repetitions (e.g., 4-5) and wider velocity-loss threshold ranges (e.g., $5-10 \%)$. The reduced absolute error in the second 60\%1RM session suggests that POV accuracy can be improved with practice. The systematic underestimation error in the squat suggests that a correction factor may increase POV accuracy in this exercise.

Keywords: velocity-based training, perception, auto-regulation, estimation

\section{Introduction}

Velocity-based training (VBT) is a method used by strength and conditioning professionals to prescribe and monitor resistance training programs based on repetition velocities completed in different exercises. ${ }^{1-4}$ VBT has a number of advantages. First, a strong relationship exists between repetition velocities and both the 1 Repetition-Maximum (1RM) and the relative percentage of 1RM loads lifted in different exercises. ${ }^{5,6}$ This allows for precise estimation of the \%1RM lifted, starting from the first repetition while using submaximal loads. Second, the progressive velocity loss across repetitions within sets provides a good indication of neuromuscular fatigue ${ }^{7}$ and the number of repetitions one can complete before reaching taskfailure. ${ }^{8}$ Finally, terminating sets at certain velocity loss thresholds (e.g., 10-30\% relative to first repetition) leads to a range of positive neuromuscular adaptations. ${ }^{9-11}$ Collectively, VBT can optimize the training process by adjusting the lifted loads and the number of repetitions completed per exercise in an individualized manner. However, to benefit from VBT, certain devices that measure velocity are required, such as inertial measurement units and linear position transducers (LPT). ${ }^{12}$ While the costs of such devices decreased in recent years, they are still not affordable to many. Moreover, using velocity measuring devices with large groups 
can be an impractical task. When velocity measuring devices are unavailable or impractical, a possible alternative can be the trainee's perception of movement velocity. Assuming acceptable accuracy levels, applying VBT methodologies based on trainee's perception can be a useful and practical solution, due to its simplicity and cost-effectiveness.

To our knowledge, perception of velocity in resistance-training exercises was only examined in two studies by Bautista et al. ${ }^{13,14}$ In both studies, resistance-trained participants lifted loads ranging from $20 \%$ to $70 \%$ of $1 \mathrm{RM}$ in a blindfold manner for 2 to 4 repetitions, in the squat ${ }^{14}$ and bench-press ${ }^{13}$ exercises. Participants reported the perceived mean bar velocity at the completion of each set using a velocity scale ranging from 0.1 to $1.6 \mathrm{~m} / \mathrm{s}^{-1}$, that is accompanied by five qualitative descriptors (e.g., "very slow" and "power zone"). Actual bar velocity was concurrently measured using a LPT. In both studies, the relationship between perceived and actual velocity was strong $(\mathrm{r}$ range $=0.88-0.99)$, indicating excellent accuracy. While the studies by Bautista et al. are an important step in the right direction, they include a number of limitations that warrant further investigations.

First, the relationship between perceived and actual velocity in Bautista's studies could be partly explained by the different loads on the bar which varied constantly and considerably between sets within the same session. Since velocity changes as a function of load, perception of heaviness in each set could have guided participants in their ratings, making the relationship stronger than if the load was constant. Second, participants reported the average velocity of each set that consisted of two to four repetitions. Since people tend to selectively remember the peak and the end of an event (i.e., peak-end rule ${ }^{15,16}$ ), it is of interest to examine velocity estimation of single repetitions, rather than sets. Third, Bautista's scale uses absolute velocity units (i.e., $\mathrm{m} / \mathrm{s}^{-1}$ ), which may be more difficult to grasp compared with other measurement 
units, such as percentages expressed relatively to a fixed anchor point. Forth, a direct comparison in estimation accuracy between the bench-press and squat was not conducted in Bautista's studies. In view of the differences between the two exercises in range of motion, muscle mass, body position, and more, a direct comparison between them within the same study is of additional interest.

Accordingly, the purpose of the present study was to complement and expand upon Bautista's work. We investigated the accuracy of perceived bar velocity among resistance-trained participants performing the barbell squat and bench-press exercises. Across three sessions, participants performed both exercises while lifting the same relative loads within a given session $(60 \%$ or $70 \%$ of $1 \mathrm{RM})$. Participants completed four sets of eight repetitions and reported their perceived bar velocity after each repetition as a percent of their perceived velocity of the first repetition. In view of Bautista's work, we expected medium to strong estimation accuracies. However, given the differences between studies, our expectations were only tentative.

\section{Materials and methods}

\section{Participants}

Twenty male collegiate sport-science students volunteered to participate in the study (Table 1). To be included in the study participants had to be healthy, between the ages of 18 and 45 , and have at least two years of resistance training experience in performing the squat and bench-press exercises. Finally, a ratio of at least 1.2 between the load lifted at 1RM and bodyweight in both exercises was required. Written informed consent was obtained after the participants received an oral explanation of the purpose and potential risks of the study. All 
procedures were conducted in accordance with the Helsinki Declaration and approved by the Institution's Ethics Committee.

\section{Procedure}

A randomized cross-over design was used to investigate the relationship between perceived and actual bar velocity during the barbell squat and bench-press exercises performed in a Smith-Machine (Technogym Equipment, Barcelona, Spain). Participants reported to the laboratory for four sessions separated by three to six days. In the first session, participants completed a 1RM test in the barbell squat followed by the bench-press and were familiarized with the experimental procedures. In the three subsequent sessions, participants followed the exact same procedures, consisting of four sets of eight repetitions of squats followed by the bench presses, with three minutes of rest between sets and exercises. In two of the experimental sessions, participants lifted $60 \%$ of 1RM in both exercises and in one session they lifted $70 \%$ of 1RM. These loads were selected as they fall within the recommended range for power development in the squat and bench-press exercises (30-70\% of 1RM). ${ }^{17,18}$ After completing each repetition, participants verbally reported their perceived bar velocity which was recorded via a tie-microphone attached to their shirts. Actual bar velocity was measured with a LPT.

\section{RM tests and familiarization (session 1)}

Anthropometric measurements were taken and followed by an assessment of the required squat depth corresponding to a $90^{\circ}$ knee angle measured with a hand-held goniometer. To ensure similar depth across sessions, a box with adjustable height was placed underneath the participants to which they were required to gently squat onto. Participants then performed a 
structured warm-up protocol consisting of dynamic stretching and calisthenics, followed by an individualized five-minutes warm-up. This warm-up protocol was identical in all sessions. Thereafter, participants were assessed in the barbell squat 1RM followed by the bench-press 1RM. The 1RM protocol consisted of consecutive lifts with progressively heavier loads until reaching the estimated or true $1 \mathrm{RM}$. Two to three minutes of rest were provided between sets once the loads reached $90 \%$ of estimated 1RM. Bar velocity was recorded in all progressive 1RM attempts.

Following the 1RM tests, participants were familiarized with the procedure of the experimental sessions by performing two sets of 8-12 repetitions with an empty barbell in both exercises. Specifically, participants were asked to verbally report their perceived bar velocity starting from the second repetition and onward as a percent of the first repetition. That is, the first repetition was always considered $100 \%$ irrespective if the actual velocity resulted in the fastest repetition or not. Then, starting from the second repetition, participants reported percent values in relation to the first one. To illustrate, if any repetition starting from the second one is perceived to be as fast as the first one, then $100 \%$ is the value that should be reported. Alternately, if any repetition is perceived to be slower or faster by $5 \%$ relative to the first one, then $95 \%$ and $105 \%$ are the values that should be reported, respectively. Participants were asked to execute the concentric phase of each repetition as fast as possible. After each completed repetition, participants were asked to verbally state their perceived bar velocity.

\section{Experimental sessions (sessions 2-4)}


Following the standard warm-up, participants completed a progressive warm-up consisting of $10,5,3,2$ repetitions loaded with an empty bar, $40 \% 50 \% 60 \%$ of $1 \mathrm{RM}$, respectively. In the $70 \%$ conditions, they completed two more reps with $70 \%$ of 1 RM. The first warm-up set was used to practice the ratings of each repetition. Following two minutes of rest, participants completed four sets of eight repetitions with either $60 \%$ or $70 \%$ of 1 RM. All sessions were performed at the same facilities, ran by the same two researchers at approximately the same time of the day ( \pm 2 hours). Participants were asked to refrain from intense training $24 \mathrm{~h}$ prior to testing days and to avoid muscular fatigue and soreness.

\section{Bar velocity data collection}

The mean propulsive velocity of bar movement during the concentric phase for each repetition in both exercises was examined. A LPT (Chronojump, Barcelona, Spain) sampling at 1000 $\mathrm{Hz}$, fixed to the bar of the Smith machine at a perpendicular angle to the floor, and the commercial software provided by the manufacturer in conjunction with the device, were used to collect and compute the bar velocity outcomes. According to the software specifications, instantaneous velocity was smoothed with a fourth-order low-pass Butterworth filter, with a cut-off frequency of $10 \mathrm{~Hz}$.

(Table 1 near here)

\section{Statistical analysis}

We descriptively inspected the individual data points using two approaches. First, we plotted the raw percentage points differences between the perceived and actual bar velocity in all repetitions, relative to the first repetition. Second, we transformed all data points to absolute 
values (i.e., ignoring direction of error) which allowed us to quantify the extent of the average error while overcoming the offsetting effect of conflicting negative and positive errors.

To inferentially analyze the absolute differences between perceived and actual decrease in velocity relative to the first repetition, we used the following model:

(1) $\mid$ Percived velocity - actual velocity $\left.\right|_{i t}=\beta_{0}+\beta_{1-2}$ session $_{i t}+\beta_{3-5}$ set $_{i t}+\beta_{6}$ repetition $_{i t}+$ $\beta_{7}$ exercise $_{i t}+\beta_{k} z_{i t}+a_{i}+\varepsilon_{i t}$

where absolute error ${ }_{i t}$ is the percentage point difference between perceived and objective bar velocity decrease from the first repetition of every set of subject $i$ at repetition $t ; a_{\mathrm{i}}$ is the subject specific deviation from the grand mean; and $\beta_{\mathrm{k}}$ represents all coefficients of three twoway interactions between exercise (squat=1) on the one hand, and sessions, sets and repetitions on the other.

Turning to the direction of the error, we defined "underestimation of bar velocity" as observations in which subjects perceived the velocity to be lower than the objective velocity $(=1)$ and contrasted these to all other cases (perceived velocity was equal to or higher than objective velocity $=0$ ). We analyzed this outcome using a conditional fixed effects logistic regression as follows:

(2) $\ln \frac{p\left(U . E_{i t}=1\right)}{p\left(U . E_{i t}=0\right)}=\beta_{1-2}$ Session $_{i t}+\beta_{3-5}$ set $_{i t}+\beta_{6}$ repetition $_{i t}+\beta_{7}$ exercise $_{i t}+\beta_{g} z_{i t}+\varepsilon_{i t}$

Where $\mathrm{U} . \mathrm{E}_{\mathrm{it}}=1$ denotes subject $i$ at repetition $t$ underestimating bar velocity; and $\beta_{\mathrm{g}}$ represents all coefficients of the three two-way interactions between exercise on the one hand, and sessions, sets and repetitions on the other. Note that $\beta_{0}$ and $\mathrm{a}_{\mathrm{i}}$ have been "conditioned" out of the likelihood function. In addition, to ease interpretation of the results and enable a simple comparison between all analyzed categories in probability of underestimation, rather than odds ratios relative to the reference category, we calculated average predicted probabilities by 
exercise and across sessions, sets and repetitions by using an unconditional fixed effects logistic regression.

\section{Results}

\section{Descriptive statistics}

Figures 1 and 2 depict the raw and absolute individual data points, respectively, across sessions, exercises and repetitions. We observed two main patterns in Figure 1. First, variance increased across repetitions in both exercises. Second, in the bench-press the mean remained relatively close to zero whereas in the squat it was consistently under the zero line, and the extent of this underestimation error grew as a function of the repetitions. In Figure 2, the increase in variance across repetitions is similar to that illustrated in Figure 1. However, the mean absolute error increased in a similar fashion in both exercises.

(Figure 1 and 2 near here)

\section{Extent of error}

In this section we focus on the key results and direct the interested reader to explore all other effects fully presented in Table 2 and visible in Figure 3. When controlling for sessions, sets, repetitions and subjects, the absolute error was 1.69 percentage points higher in the squat than in the bench-press $(\mathrm{p}<0.001)$. In both exercises, the absolute error was lower in the second $60 \% 1 \mathrm{RM}$ session compared to the first session, with significant reductions of 2.2 and 1.2 percentage points in the squat and bench-press, respectively ( $\mathrm{p} \leq 0.007)$. In both exercises, the absolute error in the 70\%1RM was mostly similar to the first $60 \% 1 \mathrm{RM}$ session ( $\mathrm{p} \geq 0.311$, $0.31<b<0.45)$, but significantly higher than the second $60 \% 1 \mathrm{RM}$ session $(\mathrm{p}<0.001, b \approx 1.7)$. We observed no meaningful differences in these effects between the exercises ( $p>0.117$, $1<\mathrm{b}<-0.8$ percentage points). 
In the bench-press, the absolute error in the third and fourth sets was higher than the first set $(\mathrm{p} \leq 0.013, \mathrm{~b} \approx 1.3)$. In contrast, in the squat, differences between the sets were trivial $(\mathrm{p} \geq 0.187$, $-0.67<b<0.3$ percentage points). Yet, excluding the effect of set $3(p=0.002, b=2.2)$, differences between the exercises in the effects of sets were negligible.

In both exercises the absolute error increased with every successive repetition. The increment in the error related with each completed repetition was significantly higher in the squat $(\mathrm{p}<0.001, \mathrm{~b}=1.8)$ compared to the bench-press $(\mathrm{p}<0.001, \mathrm{~b}=1.2)$. Therefore, while the error in both exercises in the second repetition is estimated at $\sim 5.85$ percentage-points, by the eighth repetition it increased to 13.2 in the bench-press and to 16.7 percentage-points in the squat.

(Figure 3 and Table 2 near here)

\section{Direction of error}

All effects are presented in Table 3 and visible in Figure 4. The odds of underestimating bar velocity, controlling for sessions, sets, repetitions and subjects were 4.24 times higher in the squat exercise compared with the bench-press $(\mathrm{p}<0.001)$. In the bench-press, the odds of underestimating bar velocity decreases as sessions and sets progress $(\mathrm{p}<0.001)$. In contrast, we detected no effect of session or set in the squat ( $p \geq 0.397,0.85<0 R<1.01)$. Therefore, the decrease in the odds of underestimating bar velocity were larger in the bench-press when compared with the squat in session $3(\mathrm{p}=0.012, \mathrm{OR}=1.7)$ and, likewise, in sets 3 and 4 ( $p<0.001, O R=2.31$ and 2.64, respectively). The odds of underestimating velocity in the squat increased, on average, with each repetition by $17 \%(\mathrm{p}<0.001)$. In contrast, they decreased, on average, by $30 \%$ with each repetition in the bench-press $(\mathrm{p}<0.001)$. The effect of repetition significantly differed between the exercises $(\mathrm{p}<0.001)$. 
Comparing the predicted probabilities of underestimating velocity (using unconditional maximum likelihood model), we note that in the squat the probability of underestimating velocity remained almost constant at $\sim 80 \%$ throughout sessions and sets and grew from $71 \%$ to $83 \%$ as repetitions progressed. In contrast, in the bench-press, the probability of underestimating velocity decreased from $71 \%$ in repetition 2 to $35 \%$ in repetition 8 and from $\sim 60 \%$ in the first session or set to as low as $\sim 47.5 \%$ in the last session or set.

(Figure 4 and Table 3 near here)

\section{Discussion}

The purpose of this study was to examine the accuracy of perceived bar velocity among resistance-trained participants performing the barbell squat and bench-press across loads, sets and repetitions. We observed three main findings. First, in both exercises, the absolute error rates increased with consecutive repetitions, and the growth in error was larger in the squat, compared to the bench. Second, subjects improved their accuracy ratings in the second $60 \% 1 \mathrm{RM}$ session. Third, looking at the error's direction, subjects systematically underestimated velocity in the squat across all repetitions.

Across exercises a similar pattern emerged, in which the absolute error increased with consecutive repetitions. Specifically, the absolute error increased by 1.2 percentage points in the bench-press and by 1.8 in the squat. These errors can be explained by a number of reasons. The lack of calibration against the actual velocity of repetitions may have hindered participant's accuracy levels. We assume that informing participants about the extent of their

error in real time can improve estimation accuracy. ${ }^{19}$ This assumption is supported by the fact that participants improved estimation accuracy from the first to the second 60\%1RM session 
in both exercises, despite not receiving any information about actual velocity. Since all ratings were based on participant's perception of bar velocity of the first repetition, miscalculating the velocity of this anchor could have profoundly impacted the remaining ratings during the ongoing set. Then, the time elapsed between the first and subsequent repetitions could have altered the perception of the velocity in the first repetition, which could further hinder estimation accuracy. Finally, the neuromuscular fatigue that had likely accumulated with progressive repetitions (e.g., lactate) may have interfered with the ability to accurately estimate movement velocity. ${ }^{20,21}$ As a whole, these findings are inconsistent with Bautista work, ${ }^{13,14}$ in which accurate velocity estimation values were observed. These differences likely stem from the large differences in designs and measurement strategies which also preclude a direct comparison.

The larger absolute errors observed in the squat compared to the bench-press (1.7 percentagepoints differences), can result from a number of differences between the exercises. The mean distance the barbell travelled was 0.35 meters in the bench-presses and 0.55 meters in the squats. The bench-press is also characterized by a greater propulsive portion of the bar compared to the squat. ${ }^{22,23}$ The shorter distances the barbell travels in the bench-press, coupled with its longer propulsive phase, may have facilitated a more accurate perception of its velocity. Then, in contrast to the squat, during the bench-press participants were able to visually observe parts of the moving bar which may have assisted in gauging its velocity. The amount and size of muscle mass involved in the squat exercise is greater than the bench-press. Finally, upper body muscles are used more frequently than the lower body muscles during daily activities, ${ }^{24}$ and are commonly involved in fine motor tasks requiring movement accuracy, such as grasping and reaching. This fact can also explain why movement velocity 
with the upper body is estimated with greater precision. Future studies are required to directly examine the posed explanations to the differences between the body parts.

In contrast to the absolute error, a divergent pattern emerged between the exercises when inspecting the directional error. Large and systematic underestimation of bar velocity errors occurred in the squat. Conversely, a smaller shift from over- to underestimation of bar velocity error occurred in the bench-press. Collectively, participants were 4.2 times more likely to underestimate barbell velocity in the squat compared with the bench-press. While some of the speculative reasons mentioned previously concerning the differences between exercises in absolute error may also hold true for the directional error, we are uncertain why such large differences between exercises occurred. Specifically, we are uncertain what can cause the systematic underestimation in the squat. Practically, if using perception of bar velocity to reach a velocity threshold in the squat, it may be reasonable to use a correction factor to overcome the systematic bias.

This study has a number of strengths and limitations worthy of discussion. In total, more than 3300 repetitions were collected and analyzed, adding considerable statistical power to this study. While the participants in this study were experienced in resistance-training, they were not experienced in VBT, and were not presented with actual bar velocity throughout the study. Future studies are required to examine if perception of velocity is more accurate among those experienced with VBT, and studies that include actual bar velocity information to examine if, and to what extent, estimation accuracy can be improved. Additionally, developing a richer understanding of the underlining mechanisms causing differences between upper and lower body exercises is warranted. 


\section{Practical Application}

In view of the extent of the absolute error across exercises, terminating sets based on perception may lead to suboptimal results in sets that require a high number of repetitions coupled with low and precise velocity loss thresholds. Accordingly, perception of velocity may be more applicable if used to guide one through sets that include fewer repetitions (e.g., up to five) and tentative velocity loss threshold ranges (e.g., 5\% to 10\%). The reduced error rates observed in the second 60\%1RM session in both exercises suggests that accuracy rates can improve with practice. Finally, since subjects underestimated velocity in the squat, a correction factor may improve accuracy in this exercise. For example, if the goal is a velocity loss of $10 \%$ per set and the expected underestimation is $5 \%$, then requiring one to terminate a set once the perceived velocity loss corresponds to $15 \%$ may lead to a more accurate result.

\section{Conclusion}

To our knowledge, this is the first study to investigate the accuracy levels of perception of velocity as a percent of the first repetition in the squat and bench-press, across loads, sets, and repetitions. We observed that the absolute error increased with subsequent repetitions in both exercises, with larger errors in the squat. Participants improved their accuracy ratings between the first and second 60\%1RM session across exercises. A systematic bias was observed in the squat, in which participants underestimated actual bar velocity. No meaningful effect of sets and load occurred. Given the potential of this approach, more research is currently required to examine the estimation accuracy levels of different populations and the extent of possible improvements in estimation accuracy.

\section{Conflict of interests}


MS, AL, ADI and IH declare no conflict of interests.

\section{References}

1. Guerriero A, Varalda C, Piacentini MF. The Role of Velocity Based Training in the Strength Periodization for Modern Athletes. Journal of Functional Morphology and Kinesiology. 2018;3(4):55-62.

2. Jovanović M, Flanagan EP. Researched applications of velocity based strength training. Journal of Australian Strength and Conditioning. 2014;22(2):58-69.

3. Nevin J. Autoregulated Resistance Training: Does Velocity-Based Training Represent the Future? Strength and Conditioning Journal. 2019;41(4):34-39.

4. Mann JB, Ivey PA, Sayers SP. Velocity-based training in football. Strength and Conditioning Journal. 2015;37(6):52-57.

5. Conceição F, Fernandes J, Lewis M, Gonzaléz-Badillo JJ, Jimenéz-Reyes P. Movement velocity as a measure of exercise intensity in three lower limb exercises. Journal of Sports Sciences. 2016;34(12):1099-1106.

6. Jidovtseff B, Harris NK, Crielaard J-M, Cronin JB. Using the load-velocity relationship for 1RM prediction. The Journal of Strength and Conditioning Research. 2011;25(1):267-270.

7. Sánchez-Medina L, González-Badillo JJ. Velocity Loss as an Indicator of Neuromuscular Fatigue during Resistance Training. Medicine and Science in Sports and Exercise. 2011;43(9):1725-1734.

8. Gonzalez-Badillo JJ, Yanez-Garcia JM, Mora-Custodio R, Rodriguez-Rosell D. Velocity Loss as a Variable for Monitoring Resistance Exercise. International Journal of Sports Medicine. 2017;38(3):217-225.

9. Pareja-Blanco F, Sánchez-Medina L, Suárez-Arrones L, González-Badillo JJ. Effects of velocity loss during resistance training on performance in professional soccer players. International Journal of Sports Physiology and Performance. 2017;12(4):512-519.

10. Pareja-Blanco F, Rodríguez-Rosell D, Sánchez-Medina L, et al. Effects of velocity loss during resistance training on athletic performance, strength gains and muscle adaptations. Scandinavian Journal of Medicine and Science in Sports. 2017;27(7):724-735.

11. Sánchez-Moreno M, Cornejo-Daza PJ, González-Badillo JJ, Pareja-Blanco F. Effects of Velocity Loss During Body Mass Prone-Grip Pull-up Training on Strength and Endurance Performance. The Journal of Strength and Conditioning Research. 2020;34(4):911-917.

12. Pérez-Castilla A, Piepoli A, Delgado-García G, Garrido-Blanca G, García-Ramos A. Reliability and concurrent validity of seven commercially available devices for the 
assessment of movement velocity at different intensities during the bench press. The Journal of Strength and Conditioning Research. 2019;33(5):1258-1265.

13. Bautista IJ, Chirosa IJ, Chirosa LJ, Martín I, González A, Robertson RJ. Development and validity of a scale of perception of velocity in resistance exercise. Journal of Sports Science \& Medicine. 2014;13(3):542.

14. Bautista IJ, Chirosa IJ, Robinson JE, Chirosa LJ, Martínez I. Concurrent validity of a velocity perception scale to monitor back squat exercise intensity in young skiers. The Journal of Strength and Conditioning Research. 2016;30(2):421-429.

15. Hargreaves EA, Stych K. Exploring the peak and end rule of past affective episodes within the exercise context. Psychology of Sport and Exercise. 2013;14(2):169-178.

16. Kahneman D, Fredrickson BL, Schreiber CA, Redelmeier DA. When more pain is preferred to less: Adding a better end. Psychological Science. 1993;4(6):401-405.

17. Soriano MA, Jimenez-Reyes P, Rhea MR, Marin PJ. The Optimal Load for Maximal Power Production During Lower-Body Resistance Exercises: A Meta-Analysis. Sports Medicine. 2015;45(8):1191-1205.

18. Soriano MA, Suchomel TJ, Marin PJ. The Optimal Load for Maximal Power Production During Upper-Body Resistance Exercises: A Meta-Analysis. Sports Medicine. 2017;47(4):757-768.

19. Lauber B, Keller M. Improving motor performance: Selected aspects of augmented feedback in exercise and health. European Journal of Sport Science. 2014;14(1):3643.

20. Forestier N, Teasdale N, Nougier V. Alteration of the position sense at the ankle induced by muscular fatigue in humans. Medicine and Science in Sports and Exercise. 2002;34(1):117-122.

21. Ju Y-Y, Wang C-W, Cheng H-YK. Effects of active fatiguing movement versus passive repetitive movement on knee proprioception. Clinical Biomechanics. 2010;25(7):708-712.

22. Pérez-Castilla A, García-Ramos A, Padial P, Morales-Artacho AJ, Feriche B. Loadvelocity relationship in variations of the half-squat exercise: Influence of execution technique. The Journal of Strength and Conditioning Research. 2020;34(4):10241031.

23. Sanchez-Medina L, Perez C, Gonzalez-Badillo J. Importance of the propulsive phase in strength assessment. International Journal of Sports Medicine. 2010;31(02):123129.

24. Kern DS, Semmler JG, Enoka RM. Long-term activity in upper-and lower-limb muscles of humans. Journal of Applied Physiology. 2001;91(5):2224-2232. 


\section{Figure captions}

Figure 1. Individual data points of the percentage-points differences between perceived and actual bar velocity across sets. The mid-horizontal line and error bars represent mean and SDs.
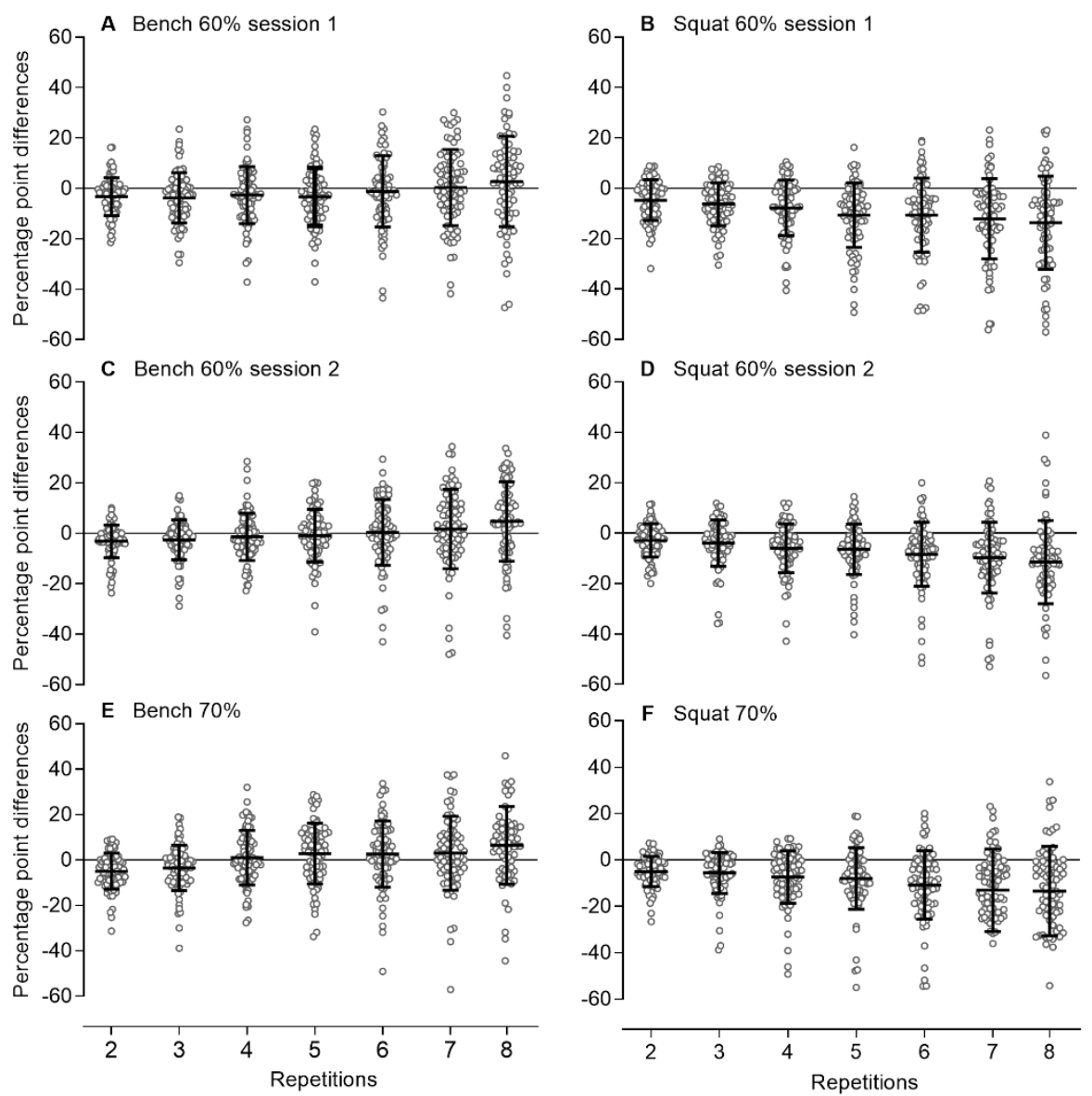
Figure 2. Individual absolute data points (i.e., ignoring the direction of error) of the percentage-points differences between perceived and actual bar velocity across sets. The mid-horizontal line and error bars represent mean and SDs.
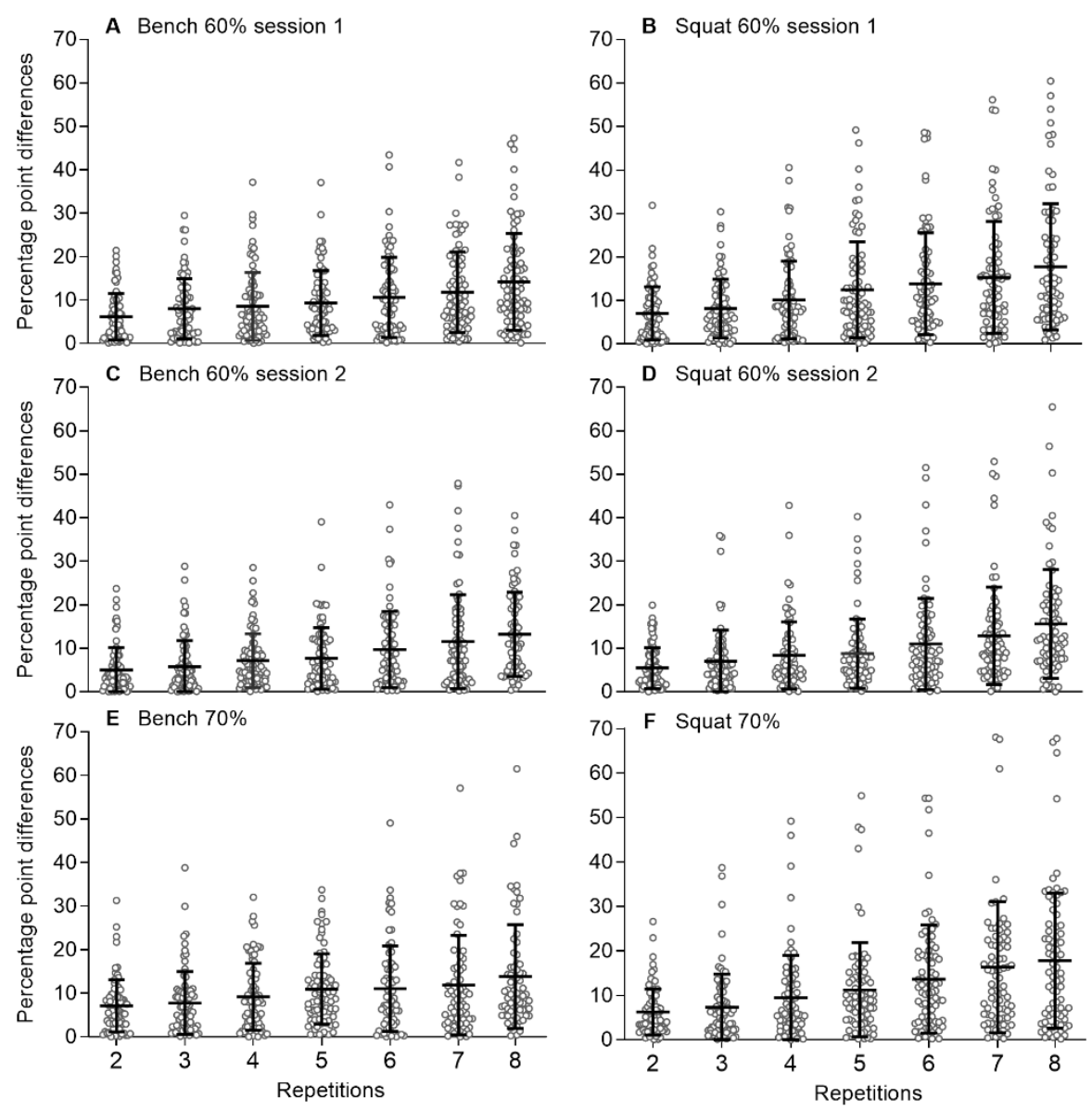
Figure 3. The graphs represent the absolute error across sessions (A), sets (B), and repetitions $(\mathrm{C})$. The circles and error bars represent means and $95 \%$ confidence intervals.

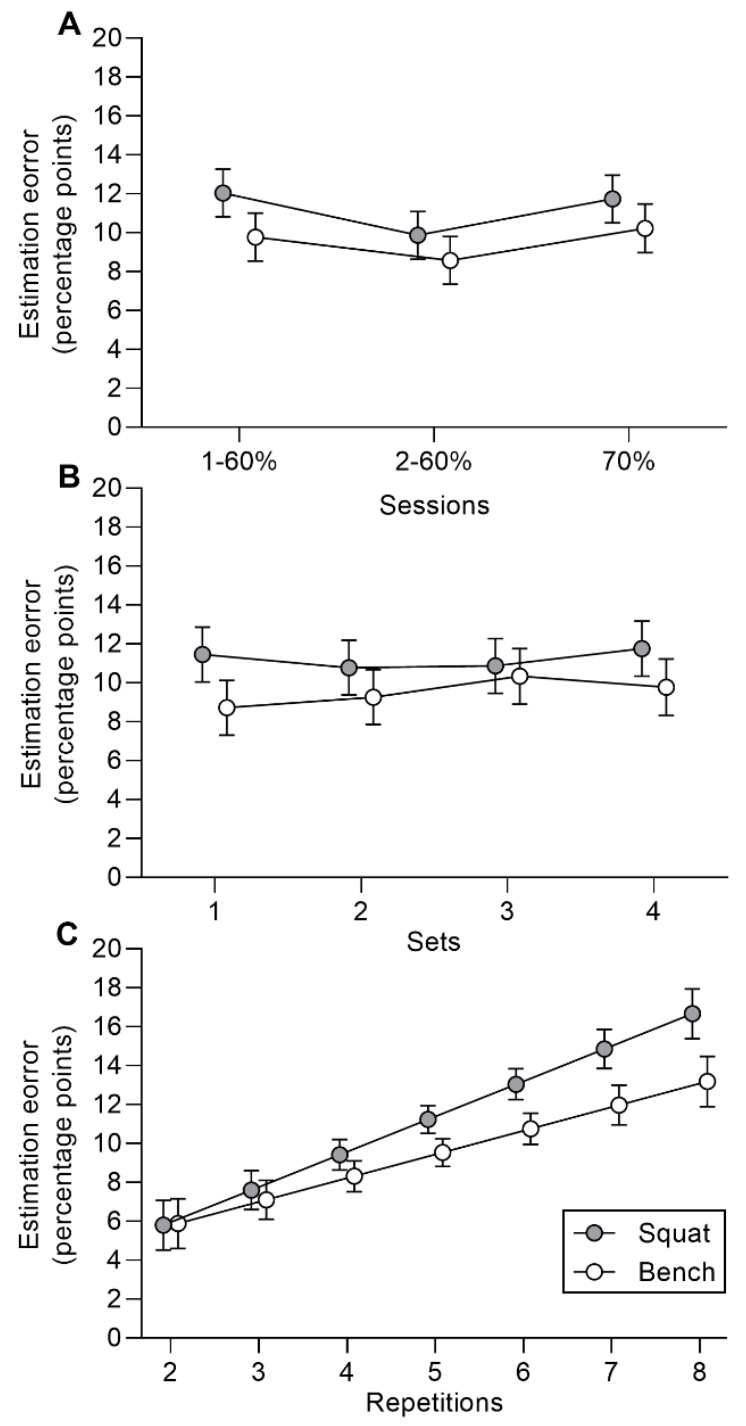


Figure 4. The graphs represent the probability of underestimating actual velocity across sessions (A), sets (B), and repetitions (C). The circles and error bars represent means and 95\% confidence intervals. The dashed horizontal line represents no directional error.

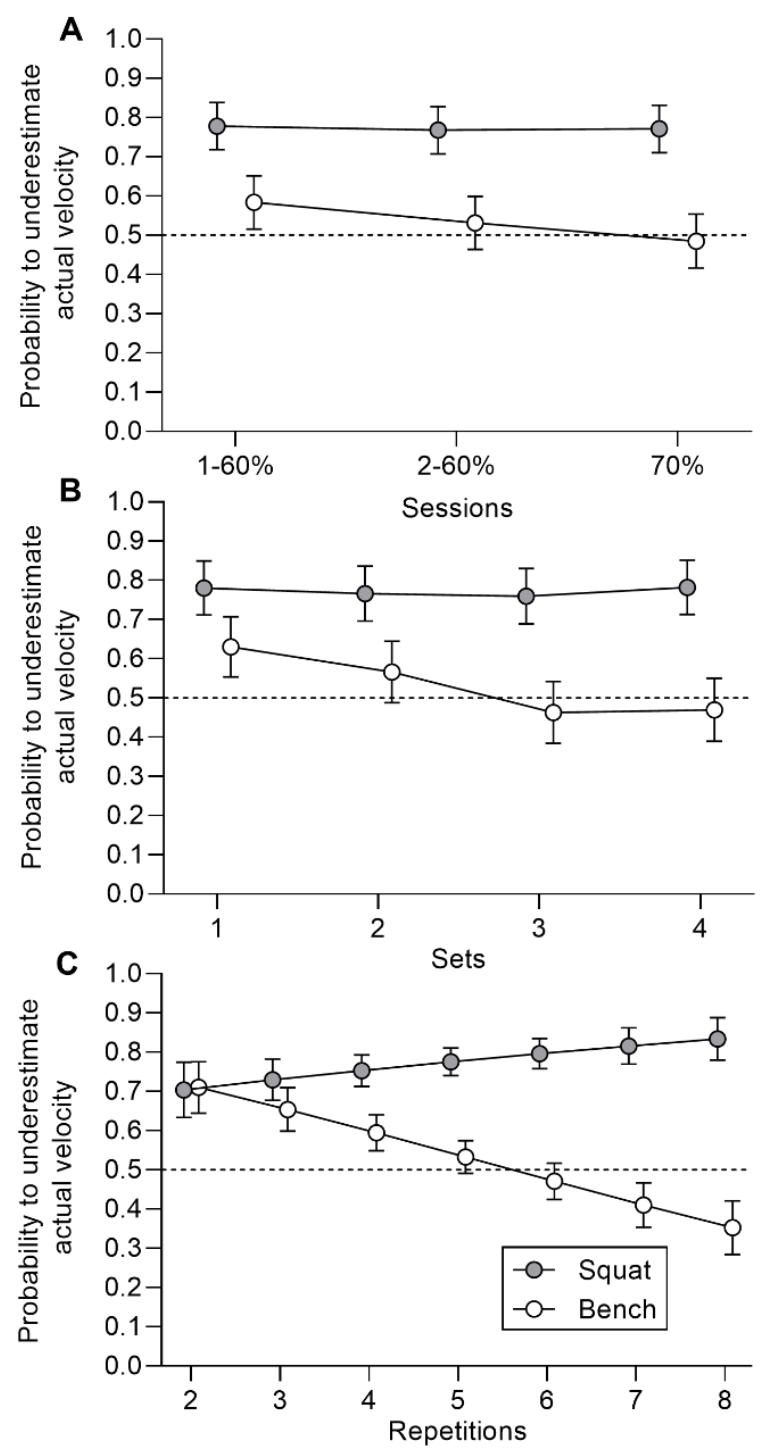


Table 1. General Demographics.

\begin{tabular}{l|c}
\hline & $\begin{array}{c}\text { Mean } \pm \text { SD } \\
\text { (Range) }\end{array}$ \\
\hline Age & $25.8 \pm 2.4$ \\
& $(23-31)$ \\
\hline Height $(\mathrm{cm})$ & $175 \pm 6$ \\
& $(163-188)$ \\
\hline Weight $(\mathrm{kg})$ & $79.7 \pm 10$ \\
& $(65-100)$ \\
\hline Experience in RT (yrs) & $5.9 \pm 3.7$ \\
& $(2-15)$ \\
\hline Mean workouts per week & $4.5 \pm 0.5$ \\
& $(4-5)$ \\
\hline 1RM barbell bench-press & $112.5 \pm 18.5$ \\
(kg) & $(73-145)$ \\
\hline 1RM/ Bodyweight & $1.42 \pm 0.2$ \\
bench press & $(1-1.82)$ \\
\hline Average velocity 1RM & $0.14 \pm 0.04$ \\
barbell bench press (m·s $\left.{ }^{-1}\right)$ & $(0.08-0.23)$ \\
\hline 1RM barbell squat (kg) & $130.9 \pm 21.3$ \\
& $(90-182)$ \\
\hline 1RM/ Bodyweight squat & $1.64 \pm 0.2$ \\
& $(1.2-2.1)$ \\
\hline Average velocity 1RM & $0.25 \pm .06$ \\
barbell squat (m·s $\left.\mathrm{s}^{-1}\right)$ & $(0.18-0.36)$ \\
\hline 1RM - one repetition maximum; RT - \\
resistance training; SD - standard \\
deviation & \\
& \\
\hline
\end{tabular}


Table 2. Fixed effects (within subjects) linear regression of extent of error in velocity

\begin{tabular}{lcc}
\hline & $\underline{\mathbf{b}}$ & $\underline{\mathbf{S . E}}$ \\
Constant & $5.307 * * *$ & 1.562 \\
\hline [Session 60\%A] & & \\
Session 60\%B & $-1.196 * *$ & 0.442 \\
Session 70\% & 0.45 & 0.446 \\
\hline [Set 1] & & \\
Set 2 & 0.545 & 0.509 \\
Set 3 & $1.616 * *$ & 0.51 \\
Set 4 & $1.064 *$ & 0.516 \\
\hline Repetitions & $1.217 * * *$ & 0.0909 \\
\hline [Bench-press] & & \\
Squat & $1.544 *$ & 0.733 \\
\hline Session 60\%B*Squat & -0.981 & 0.625 \\
Session 70\%*Squat & -0.765 & 0.628 \\
\hline Set 2*Squat & -1.216 & 0.719 \\
Set 3*Squat & $-2.198 * *$ & 0.72 \\
Set 4*Squat & -0.759 & 0.726 \\
\hline Repetitions*Squat & $0.594 * * *$ & 0.128 \\
\hline \hline N (within 20 subjects) & \multicolumn{2}{c}{3,324} \\
\hline$*$ p $<0.05 ; * *$ p $<0.01 ; * *$ p $<0.001$ \\
\hline
\end{tabular}


Table 3. Conditional fixed-effects logistic regression of odds of underestimating bar velocity

\begin{tabular}{|c|c|c|}
\hline & $\frac{\text { Odds }}{\underline{\text { ratio }}}$ & $\underline{\text { S.E }}$ \\
\hline \multicolumn{3}{|l|}{ [Session 60\%A] } \\
\hline Session $60 \% \mathrm{~B}$ & $0.731 *$ & 0.108 \\
\hline Session $70 \%$ & $0.555 * * *$ & 0.0827 \\
\hline \multicolumn{3}{|l|}{ [Set 1] } \\
\hline Set 2 & $0.680 *$ & 0.116 \\
\hline Set 3 & $0.370 * * *$ & 0.0635 \\
\hline Set 4 & $0.385 * * *$ & 0.0668 \\
\hline Repetitions & $0.703 * * *$ & 0.0223 \\
\hline \multicolumn{3}{|l|}{ [Bench-press] } \\
\hline Squat & $0.431 * *$ & 0.113 \\
\hline $\begin{array}{l}\text { Session } \\
60 \% \mathrm{~B} * \text { Squat }\end{array}$ & 1.266 & 0.279 \\
\hline Session $70 \% *$ Squat & $1.713 *$ & 0.38 \\
\hline Set $2 *$ Squat & 1.321 & 0.336 \\
\hline Set $3 *$ Squat & $2.304 * *$ & 0.587 \\
\hline Set $4 *$ Squat & $2.634 * * *$ & 0.682 \\
\hline Repetitions*Squat & $1.672 * * *$ & 0.078 \\
\hline $\begin{array}{l}\mathrm{N} \text { (within } 20 \\
\text { subjects) }\end{array}$ & \multicolumn{2}{|c|}{3,324} \\
\hline
\end{tabular}

